\title{
AN INTRODUCTION TO F-GRAPHS, A GRAPH-THEORETIC REPRESENTATION OF NATURAL NUMBERS
}

\author{
E.J. FARRELL \\ Department of Mathematics \\ The University of the West Indies \\ St. Augustine, Trinidad \\ (Received April 4, 1987 and in revised form June 26, 1990)
}

\begin{abstract}
A special type of family graphs ( $F$-graphs, for brevity) are introduced. These are cactus-type graphs which form infinite families under an attachment operation. Some of the characterizing properties of $F$-graphs are discussed. Also, it is shown that, together with the attachment operation, these families form an infinite, commutative semigroup with unit element. Finally, it is shown that $F$-graphs are graph-theoretical representations of natural numbers.
\end{abstract}

KEY WORDS AND PHRASES. Cactus type graphs, core, pattern recognition, semigroup, semigroup isomorphism, natural numbers, ancestor (graph), parent (graph), descendant (graph), Fgraph, attaching a graph, basis graph, root of an $F$-graph.

1991 AMS SUBJECT CLASSIFICATION CODE. 05C99, $20 \mathrm{M} 07$.

\section{INTRODUCTION.}

First of all we give some definitions relative to the material which follows. For the standard definitions in Graph Theory, we refer the reader to Harary [1].

Let $(G, u)$ and $(H, v)$ be two graphs, rooted at $u$ and $v$ respectively. By attaching $\underline{H}$ to $\underline{G}$, we mean the identification of the roots $u$ and $v . H$ is therefore a subgraph of the new graph formed by the process. Suppose that we attach an isomorph of $(H, u)$ to every node of $G$, then the graph formed by doing so, is denoted by $G(H)$. Notice that if all the nodes of $H$ are equivalent (for example if $H$ is a cycle), then we speak about $G(H)$ without specifying a root in $H$. Also, since every node of $G$ is used in forming $G(H)$, it is unnecessary to speak about a root in $G$.

Let us begin with a single node $G^{0}$, then attach to it a rooted graph $(H, u)$, to form the graph $G^{1} \cong G^{0}(H)(\cong H)$. $G^{2}$ is the graph formed by attaching an isomorph of $H$ to every node of $G^{1}$ i.e., $G^{2} \cong G^{1}(H)$. In general, $G^{i+1} \cong G^{i}(H)$. By continuing in this manner, we obtain a family $F=\left\{G^{0}, G^{1}, G^{2}, \ldots\right\}$ of graphs. If $H$ is a node, then $F=\left\{G^{0}\right\}$; otherwise $F$ is infinite. We call $G^{0}$ the core of the family. $G^{1}$ is the basis of $F . G^{2}$ is the (direct) descendant of $G^{1}$ (In general, $G^{i+k}(k>0)$ is a descendant of $G^{i}$, the (immediate) parent of $G^{2}$ (In general, $G^{i}$ is an ancestor of $\left.G^{i+k}, k>0\right)$. We define the elements of $F$ to be $F$-graphs. $G^{0}$ and $G^{1}$ are called, trivial $F$-graphs. We note that $F$-graphs are a special kind of "cactus-type" graphs and are also Husimi trees (Harary [2]). For any $F$-graph $G^{k}$, we call the subgraphs isomorphic to the basis $G^{1}$, leaves of $G^{k}$. 
In the material which follows, we derive some characterizing properties of $F$-graphs, and then investigate some of the algebraic properties of the families. We show that operations can be defined on $F$-graphs, so as to create a system which is isomorphic to the system of natural numbers.

2. SOME CHARACTERIZING PROPERTIES OF F-GRAPHS.

Given an arbitrary graph $G$, it is of interest to determine (i) whether or not $G$ is a non-trivial $F$-graph and (ii) if it is, then what is its position in the family hierarchy. First of all, if $G$ is not a cactus-type graph, then it cannot be a non-trivial $F$-graph. Also, from the definition of an $F$-graph, $G$ must consist of isomorphic leaves. This criteria will eliminate many graphs. However, many cactus-type graphs will qualify, so the problem is certainly not a trivial one. For example, is the following graph $T$ a non-trivial $F$-graph?



Figure 1

The following theorem helps to characterize $F$-graphs.

THEOREM 1. Let $F=\left\{G^{0}, G^{1}, G^{2}, \ldots\right\}$ be a family of $F$-graphs in which $G^{1}$ has $m(>1)$ nodes and $n$ edges. Then $G^{r}(r>0)$ has

(i) $m^{r}$ nodes

(ii) $n\left(\frac{m^{r}-1}{m-1}\right)$ edges

(iii) $\frac{m^{r}-1}{m-1}$ leaves

PROOF. The result can be easily proved.

Theorem 1 provides a (not too useful) necessary condition for a graph to be a non-trivial $F$ graph. Since $G^{0}$ is always a node, then clearly, the members of the family are totally characterized by the (rooted) leaf $G^{1}$. If one is given a non-trivial $F$-graph, then Theorem 1 could be used to find its position $r$, only if a leaf can be determined. The determination of the leaf of an arbitrary nontrivial $F$-graph is in practice, a difficult exercise. The first inclination is to find symmetries in the graph; but this is forbidding, even in reasonably small $F$-graphs. Thus, a practical use of Theorem 1 as a necessary condition, poses great problems, since $m$ and $n$ depend on one's ability to identify the leaf.

We now refer the reader to the graph $T$ in Figure 1. Because $T$ is constructed from triangles, one is inclined to look for $T$ in the family of triangles. $T$ cannot be found in this family; and therefore the conclusion could be that $T$ is not a non-trivial $F$-graph. However, careful observation will show that $T$ consists of the graph $G^{2}$ from the family of triangles, with the leaf $G^{2}$ attached to every node using $\underline{\text { a node }}$ of valency $\underline{2} \underline{\text { as }} \underline{\text { a root. }}$. Therefore, $T$ is indeed a non-trivial $F$-graph. $T$ is the second member of the family of $F$-graphs with basis $G^{2}$ rooted at any node of valency $\underline{2}$ (i.e. the 
family of $G^{2}$ 's). It would be useful to develop some analytical means for arriving at the correct conclusion about an arbitrary $T$. At present, we are unable to do this.

3. SOME ALGEBRAIC PROPERTIES OF F-GRAPHS.

The practical problem of determining whether or not an arbitrary graph is a non-trivial $F$ graph, seems to be one of pattern recognition. In this section, we will show that the family $F$ has some beautiful algebraic properties which will have useful implications on the patterns displayed by the graphs.

In the material that follows, we assume that zero belongs to the set of natural numbers.

LEMMA 1. In $G^{r}$, there exists a node formed by the coalescence of the roots of $r$ leaves.

PROOF. This follows immediately from the construction of $G^{r}$ from $G^{0}$.

DEFINITION. Let $x$ be a node of $G^{r}$ defined by Lemma 1 . Then $x$ is a root of $G^{r}$. (Notice that it is possible for several nodes of $G^{r}$ to qualify as a root even if $G^{r}$ is not regular. Therefore any of them can be used in the attachment process). The idea of a root and the existence of a root of $G^{r}$ (by definition) are crucial to the theory of $F$-graphs. We show later on, that they are vital to the establishment of the properties of $F$-graphs.

LEMMA 2: $G^{k}=G^{k-1}\left(G^{1}\right)=G^{1}\left(G^{k-1}\right)$, for all $k>0$.

PROOF. We will prove the result by induction on $k$. For $k=1, G^{k-1}$ is $G^{0}$, which is a node. Therefore, $G^{k-1}\left(G^{1}\right)=G^{0}\left(G^{1}\right)=G^{1}=G^{1}\left(G^{0}\right)$. Let us assume that the result holds for $k-1$. Then

$$
G^{k-1}=G^{k-2}\left(G^{1}\right)=G^{1}\left(G^{k-2}\right)
$$

Now

$$
G^{k}=G^{k-1}\left(G^{1}\right)=\left(G^{1}\left(G^{k-2}\right)\right)\left(G^{1}\right) .
$$

The graph $\left(G^{1}\left(G^{k-2}\right)\right)\left(G^{1}\right)$ is obtained by attaching $G^{1}$ to every node of $G^{1}\left(G^{k-2}\right)$. In particular, $G^{1}$ is attached to every node of $G^{k-2}$. It can be seen that the roots of each $G^{k-2}$ now become the roots of each $G^{k-1}$. Hence we obtain $G^{k-1}$ attached to $G$ i.e. $G^{1}\left(G^{k-1}\right)$. Therefore,

$$
G^{k}=G^{k-1}\left(G^{1}\right)=G^{1}\left(G^{k-1}\right) .
$$

Hence, the result holds for $k$. By the Principle of Induction, it holds for all $k>0$.

Lemma 2 suggests that it does not matter whether we attach $G^{k-1}$ to $G^{1}$ or $G^{1}$ to $G^{k-1}$, when forming $G^{k}$. This idea is generalized by the following theorem.

THEOREM 2: $G^{k+s}=G^{k}\left(G^{s}\right)=G^{s}\left(G^{k}\right)$.

PROOF. We will prove the result by induction on $k$. For $k=0, G^{k}$ is a node, and the result follows trivially. For $k=1$, the result holds, as shown above in Lemma 2. Let us assume that the result holds for $k-1$. Then

$$
G^{k+s-1}=G^{k-1}\left(G^{s}\right)=G^{s}\left(G^{k-1}\right) .
$$

Clearly then,

$$
G^{k+s}=G^{k+s-1}\left(G^{1}\right)=\left(G^{k-1}\left(G^{s}\right)\right)\left(G^{1}\right)=\left(G^{s}\left(G^{k-1}\right)\left(G^{1}\right) .\right.
$$

The graph $\left(G^{k-1}\left(G^{s}\right)\right)\left(G^{1}\right)$ is the graph obtained by attaching $G^{1}$ to every node of $G^{k-1}\left(G^{s}\right)$. Now the attachment of $G^{1}$ to every node of the subgraphs $G^{s}$ of $G^{k-1}\left(G^{s}\right)$, will create subgraphs, each of which is $G^{s+1}$. Therefore, the resulting graph can be described as $G^{k-1}$ with $G^{s+1}$ attached i.e. $G^{k-1}\left(G^{s+1}\right)$. 
Consider any roof $x$ of $G^{k-1}$. The graphs with $x$ as a common node can be described as $G^{k-1}\left(G^{1}\left(G^{s}\right)\right)$ i.e. $G^{k-1}$ with the graph $G^{1}\left(G^{s}\right)$ attached to it, at $x$. But the leaf $G^{1}$ can be considered as being attached to $G^{k-1}$, and each node of $G^{1}$ has $G^{s}$ attached to it (at its root $x$ ). Also, every other node of $G^{k-1}\left(G^{1}\right)$ will have $G^{s}$ attached. Therefore, the graph $G^{k-1}\left(G^{s+1}\right)$ is also

$$
\left(G^{k-1}\left(G^{1}\right)\right)\left(G^{s}\right)=G^{k}\left(G^{s}\right)
$$

Similarly, we can show that $G^{k+s}=G^{s}\left(G^{k}\right)$ by using $G^{k+s}=G^{1}\left(G^{k+s-1}\right)$. Hence, the results holds for $k$. The proof is completed by the Principle of Induction.

Theorem 2 has some interesting implications about the description of an $F$-graph as a pattern. The graph $G^{n}$ can be described as $G^{r}$ with $G^{s}$ attached, for any pair of nonnegative integers $r$ and $s$, such that $r+s=n$. Also, we construct $G^{n}$ by attaching $G^{r}$, to $G^{s}$, for any $r, s \geq 0$, such that $r+s=n$.

DEFINITION. We define equality in $F$, as a graph isomorphism. Addition $(+)$ in $F$ is defined as follows:

$$
G^{r}+G^{s}=G^{r}\left(G^{s}\right), \text { for all } G^{r}, G^{s} \in F .
$$

It can be easily shown that + is well-defined on $F$.

COROLLARY 2.1 (Closure and Commutativity)

For all nonnegative integers $k$ and $s$, and for all $G^{k}$ and $G^{s}$ in $F$

$$
G^{k+s}=G^{k}\left(G^{s}\right)=G^{s}\left(G^{k}\right)
$$

PROOF. The result is immediate from the theorem.

LEMMA 3 (Associativity)

$$
G^{q}+\left[G^{r}+G^{s}\right]=\left[G^{q}+G^{r}\right]+G^{s} \text {, for all } G^{q}, G^{r} \text { and } G^{s} \text { in } F \text {. }
$$

PROOF: $G^{q}+\left[G^{r}+G^{s}\right]=G^{q}+G^{r+s}=G^{q+r+s}=G^{q+r}+G^{s}$

$$
=\left[G^{q}+G^{r}\right]+G^{s}, \text { for all } q, r, s \geq 0 .
$$

The associative property of + , implies that the order in which ancestors are added in the construction of a descendant, is unimportant. We can begin with any ancestor and attach the others, in any order that we choose.

Since $G^{0}$ is a node, the following result is immediate.

LEMMA 4 (Identity). Let $F$ be a family of $F$-graphs. Then

$$
G^{0}+G^{r}=G^{r}+G^{0}=G^{r}, \text { for all } G^{r} \in F .
$$

The following theorem sums up the results of Theorem 2 and Lemmas 3 and 4.

THEOREM 3. $(F,+)$ is a commutative semigroup with identify.

Let us use the symbols < and >, for the relations "is an ancestor of" and "is a descendant of" , respectively. Then for every family $F$, the following result holds.

LEMMA 4 (Trichotomy). For any two elements $G^{r}$ and $G^{s}$ in $F$, one and only one of the following holds either $G^{r}<G^{s}, G^{r}>G^{s}$ or $G^{r}=G^{s}$.

PROOF. The result follows immediately from the definitions of ancestor and descendant.

Let us define a mapping $\phi$ from $(F,+)$ to the set $N$ of natural numbers under addition, as follows:

$$
\phi\left(G^{r}\right)=r
$$


Then for all $G^{r}, G^{s} \in F$,

$$
\phi\left(G^{r}+G^{s}\right)=\phi\left(G^{r+s}\right)=r+s=\phi\left(G^{r}\right)+\phi\left(G^{s}\right) .
$$

Therefore $\phi$ is a semigroup homomorphism. Suppose that $\phi\left(G^{r}\right)=\phi\left(G^{s}\right)$. Then $r=s$, which implies that $G^{r}=G^{s}$. Therefore $\phi$ is injective. $\phi$ is clearly surjective. Therefore, $\phi$ is a semigroup isomorphism. Hence, we have the following theorem.

THEOREM $4:(F,+) \cong(N,+)$.

Theorem 4 is the crucial result for completing the equivalence between $F$ and $N$. Now we can make parallel definitions in $F$ in terms of + , by looking at definitions in $N$. For example, we define multiplication in $F$ as follows:

$$
\begin{aligned}
G^{r} \cdot G^{s} & =G^{r}+G^{r}+G^{r}+\ldots+G^{r}(s \text { times }) \\
& =G^{s}+G^{s}+G^{s}+\ldots+G^{s}(r \text { times })
\end{aligned}
$$

The roles of $G^{0}$ and $G^{1}$ as equivalent to 0 and 1 remain intact, as shown below.

$$
G^{0} \cdot G^{r}=G^{0}+G^{0}+\ldots+G^{0}(r \text { times })=G^{0}
$$

and

$$
G^{1} \cdot G^{r}=G^{1}+G^{1}+\ldots+G^{1}(r \text { times })=G^{r}
$$

Also, we have

$$
\begin{aligned}
\phi\left(G^{r} \cdot G^{s}\right. & =\phi\left(G^{r}+G^{r}+\ldots+G^{r}\right)=\phi\left(G^{r}\right)+\phi\left(G^{r}\right)+\ldots+\phi\left(G^{r}\right) \quad(s \text { times }) \\
& =r+r+r \ldots+r(s \text { times })=r s=\phi\left(G^{r}\right) \phi\left(G^{s}\right) .
\end{aligned}
$$

The following theorem can be easily established in a manner similar to that of Theorem 3 .

THEOREM 5. $(F,+)$ is a commutative semigroup with identity. From our observations on $\phi$ above, we can easily establish the following extension of Theorem 4 .

THEOREM 6: $(F,+, \cdot) \cong(N,+, \cdot)$.

The equivalence of $F$ and $N$ is now complete. We state this result in a different manner in the following corollary.

COROLLARY 6.1. F-graphs are graph-theoretic representations of natural numbers.

\section{REFERENCES}

1. HARARY, F., "Graph Theory", Addison-Wesley, Reading, Mass., 1969.

2. HARARY, F. and PALMER, E., "Graphical Enumeration", Acad. Press, New York and London, 1973. 




Advances in

Operations Research

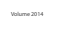



\section{The Scientific} World Journal
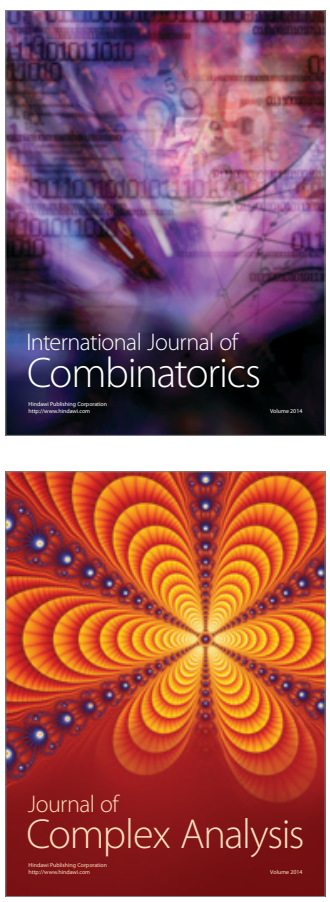

International Journal of

Mathematics and

Mathematical

Sciences
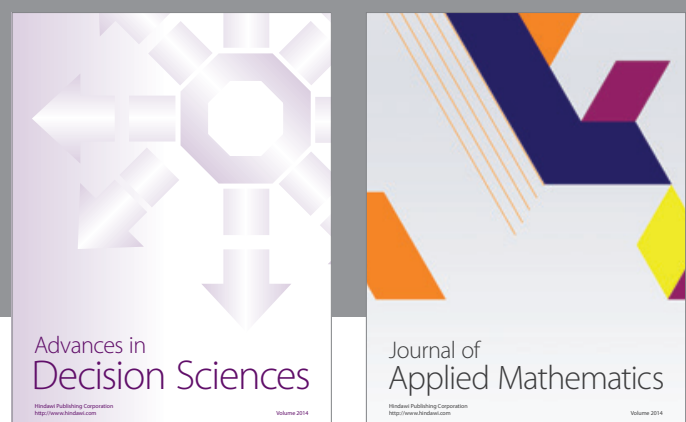

Journal of

Applied Mathematics
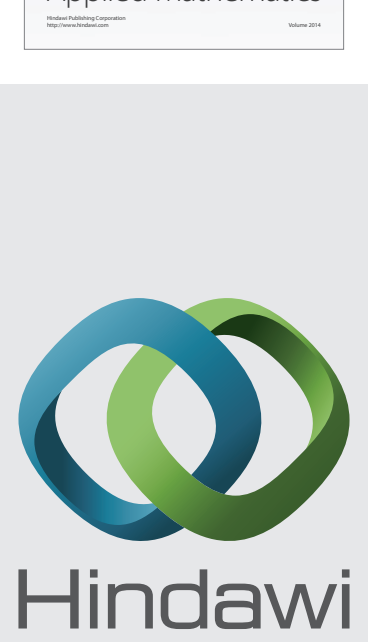

Submit your manuscripts at http://www.hindawi.com
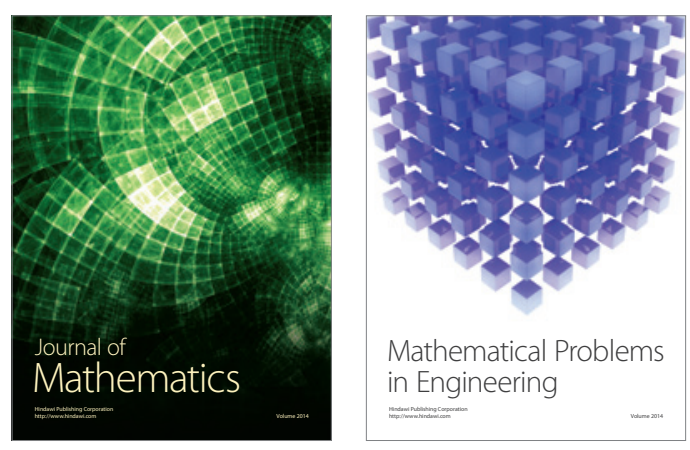

Mathematical Problems in Engineering
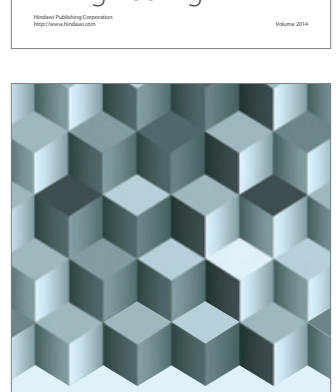

Journal of

Function Spaces
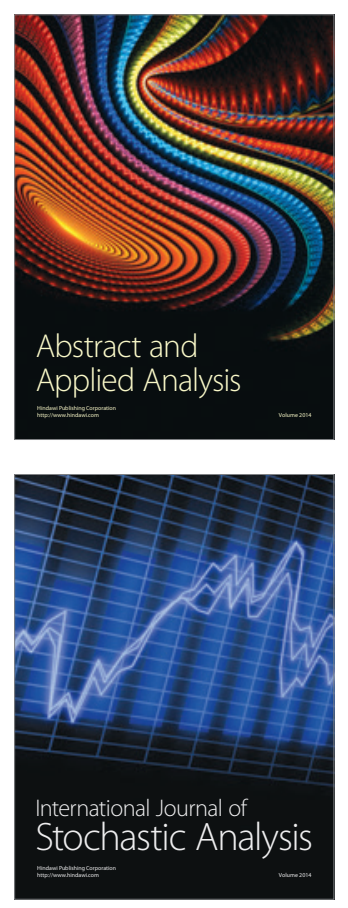

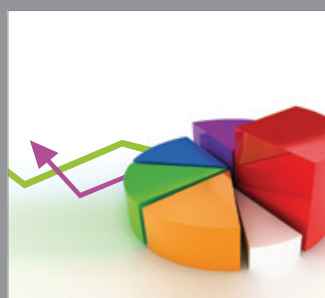

ournal of

Probability and Statistics

Promensencen
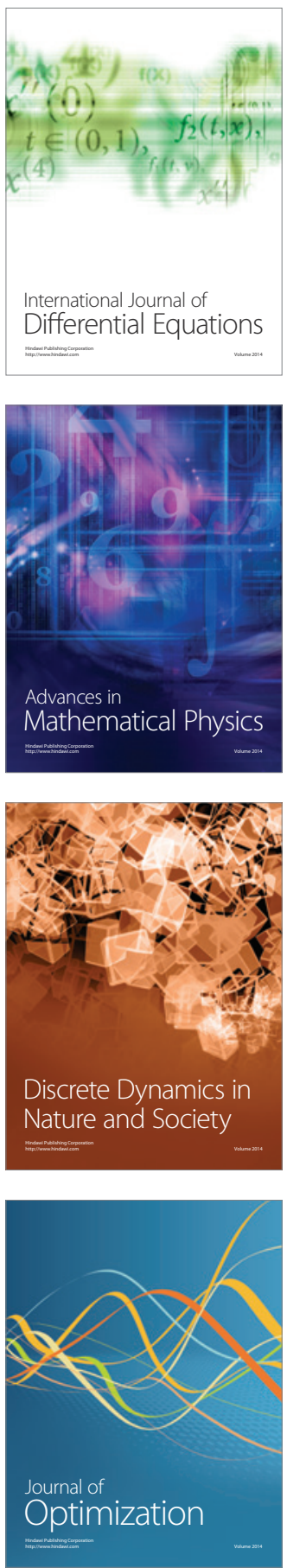